CERN-TH.7405/94

\title{
A fresh look into the heavy quark-mass values
}

\author{
S. Narison \\ Theoretical Physics Division, CERN \\ CH - 1211 Geneva 23, Switzerland \\ and \\ Laboratoire de Physique Mathématique \\ Université de Montpellier II \\ Place Eugène Bataillon \\ 34095 - Montpellier Cedex 05, France
}

\begin{abstract}
Using the recent world average $\alpha_{s}\left(M_{Z}^{2}\right)=0.118 \pm 0.006$, we give the first direct extraction from the $\Psi$ and $\Upsilon$ data of the values of the running heavy quark masses within QCD spectral sum rules to two-loops in the $\overline{M S}$-scheme: $\bar{m}_{b}\left(M_{b}^{P T 2}\right)=\left(4.23_{-0.04}^{+0.03} \pm 0.02\right) \mathrm{GeV}$ and $\bar{m}_{c}\left(M_{c}^{P T 2}\right)=\left(1.23_{-0.04}^{+0.02} \pm 0.03\right) \mathrm{GeV}$, (the errors are respectively due to $\alpha_{s}$ and to the gluon condensate), and the corresponding value of the short-distance perturbative pole masses to two-loops: $M_{b}^{P T 2}=(4.62 \pm 0.02) \mathrm{GeV}, M_{c}^{P T 2}=(1.41 \pm 0.03) \mathrm{GeV}$, which we compare with the updated values of the non-relativistic pole masses re-extracted directly from the two-loop non-relativistic sum rules: $M_{b}^{N R}=\left(4.69_{+0.02}^{-0.01} \pm 0.02\right) \mathrm{GeV}$ and $M_{c}^{N R}=$ $(1.44 \pm 0.02 \pm 0.03) \mathrm{GeV}$. It is also informative to compare the three-loop values of the short-distance pole masses: $M_{b}^{P T 3}=(4.87 \pm 0.05 \pm 0.02) \mathrm{GeV}$ and $M_{c}^{P T 3}=(1.62 \pm 0.07 \pm$ $0.03) \mathrm{GeV}$, with the dressed mass $M_{b}^{n r}=(4.94 \pm 0.10 \pm 0.03) \mathrm{GeV}$, entering into the non-relativistic Balmer formula including higher order $\alpha_{s}$ corrections. The small massdifferences $M_{b}^{N R}-M_{b}^{P T 2} \simeq M_{b}^{n r}-M_{b}^{P T 3} \simeq 70 \mathrm{MeV}$ and $M_{c}^{N R}-M_{c}^{P T 2} \simeq(30 \pm 20) \mathrm{MeV}$ can measure the size of the non-perturbative effect induced by renormalon type-singularities. An analogous analysis is pursued for the heavy-light mesons, where a simultaneous refit of the $B$ and $B^{*}$ masses from relativistic sum rules leads to: $M_{b}^{P T 2}=(4.63 \pm 0.08)$ $\mathrm{GeV}$, while the full-QCD and HQET sum rules in the large mass limit give the mesonquark mass difference to two-loops: $\left(M_{B}-M_{b}^{N R}\right)_{\infty} \simeq\left(0.6_{-0.10}^{+0.20}\right) \mathrm{GeV}$. A comparison of these new and accurate results with the existing ones in the literature is done. As a consequence, the updated values of the pseudoscalar decay constants to two-loops are: $f_{D}=(1.37 \pm 0.04 \pm 0.06) f_{\pi}$ and $f_{B}=(1.49 \pm 0.06 \pm 0.05) f_{\pi}$, which lead to $f_{B} \sqrt{B_{B}}=$ $(1.49 \pm 0.14) f_{\pi}$.
\end{abstract}

CERN-TH.7405/94

August 1994 


\section{Introduction}

Present accurate measurements of the QCD coupling $\alpha_{s}$, mainly from Z-physics and taudecay data, motivate a careful reconsideration of the existing estimates of the standard model parameters. Among others, of a prime importance is the one of the quark masses. However, unlike the electron mass, the definition of the quark masses needs a theoretical framework due to the complication implied by confinement. In particular, the notion of the pole mass from perturbation theory, though gauge independent, appears to be ambiguous, as the summation of large order perturbation theory induces a non-perturbative term due to renormalon-type singularities. However, one can still provide a good de finition of this pole mass for a truncated QCD series, which can be used consistently in a given observable known at the same order of perturbation theory. Unlike the pole mass, the running mass of the $\overline{M S}$-scheme does not suffer from this ambiguity, such that its direct estimate from the data without passing through the pole mass should be very useful.

In this paper, we shall use QCD spectral sum rules (QSSR) in order to extract, directly from the data, and for the first time, the value of the running heavy quark mass $\bar{m}_{Q}{ }^{1}$, which is a useful (and well-defined) quantity for GUTS and some other phenomenology. Indeed, many papers have been devoted in the past, either to the direct estimate of the Euclidian mass $m_{Q}\left(p^{2}=-m_{Q}^{2}\right)$ or/and of the pole mass $M_{Q}\left(p^{2}=M_{Q}^{2}\right)$ [?]-[?] while the running mass has been mainly deduced [?, ?] from its perturbative relation with the pole $\operatorname{mass}[?]^{2}$ :

$$
M_{Q}\left(M_{Q}^{2}\right)=\bar{m}_{Q}\left(M_{Q}^{2}\right)\left\{1+\frac{4}{3}\left(\frac{\alpha_{s}\left(M_{Q}^{2}\right)}{\pi}\right)+K_{Q}\left(\frac{\alpha_{s}\left(M_{Q}^{2}\right)}{\pi}\right)^{2}\right\},
$$

where $K_{b} \simeq 12.4, K_{c} \simeq 13.3$ [?]. Instead, once, we obtain the running mass from the data, we shall use the previous relation in order to deduce the short-distance perturbative pole mass $M_{Q}^{P T}$, which we shall compare with the non-relativistic pole mass $M_{Q}^{N R}$ extracted directly from the data, using non-relativistic sum rules, and with the mass $M_{Q}^{n r}$ entering into the non-relativistic Balmer formula. We shall interpret the difference between these two values as a measure of the size of the renormalon effect into the pole mass definition.

Finally, in comparing our results for the pole masses with the existing ones in the literature, we shall test the reliability of these previous results and look for the possible sources of some eventual differences between these and the ones in this paper.

\section{Input values of $\alpha_{s}$ and matching conditions}

We use the value of $\alpha_{s}\left(M_{Z}\right)$ in the range given in Table 1, where the new world average is $0.118 \pm 0.006$ [?], but we have also considered a slightly higher value of 0.127 in order to be more conservative. Then, we run this value until $M_{b}=4.6-4.7 \mathrm{GeV}$, using the

\footnotetext{
${ }^{1}$ However, an extraction of the running mass using local duality FESR has been done in [?].

${ }^{2}$ For consistency with the two-loop expression of the two-point correlator, we shall not use the threeloop relation in the sum rule analysis.
} 